\title{
STUDY OF EYE MOVEMENTS AS A RESEARCH TOOL
}

IN COGNITIVE NEUROSCIENCE

\author{
Alessandra Rufa
}

\section{Department of Neurological and Behavioural Sciences University of Siena}

\section{INTRODUCTION}

More than $60 \%$ of cerebral activity is associated with vision. Large cortical areas from the occipital to frontal regions are involved in visual processes and almost $90 \%$ of vision time is devoted to fixation. When things are apprehended by the senses, the number that can be attended to at once is small: "Pluribus intentus, minor est ad singola sensus" William James. This is especially true for the visual system, the limited perception-related informational capacity of which, is due to its neurobiological characteristics (1).

As visual information processing is relatively slow, the system has to filter overexpressed environmental information by selecting a number of relevant perceptive stimuli. The process of attention can be regarded as a sort of macroscopic filtering system. Neurophysiological and psychophysical studies have shown that visual exploration of an image does not occur in "toto", but in short spatial/temporal pieces, "minutatim", through small fixation sequences over small regions of interest. During this visual scanning, the eyes move constantly with micromovements that do not reach the level of awareness (2). When we visually scan regions of interest there is an increase in ocular micromovements providing a larger number of details of the object. Thus visual spatial attention may be considered an efficient instrument for selecting useful sensory input in online stimulus processing. Moreover, vision is a process that allows integration of small perceptual "quanti" to obtain a good representation of the visual environment. The best perception of the world is achieved when an image or a detail of interest is held steady in a specific area of the retina called the fovea. The fovea contains the highest density of photoreceptors with specific spatial and colour (wavelength) discriminating capacity. Different classes of eye movements are necessary to hold an image on the retina during head rotation or movement of the image, and to move the eye suddenly to a new point of interest in space. From a functional point of view, two major classes of eye movements are described in humans: those stabilizing gaze (optokinetic nystagmus, oculovestibular reflex) and those moving gaze (saccades, pursuits and vergence). Under natural conditions, however, a mix of all kinds of eye movements permit continuous scanning of the visual scene. The sequence of fixations and saccades during visual exploration is an expression of a number of cognitive processes; the use of standardized tasks with pre-defined spatial-temporal variables allows us to assess specific cognitive domains, such as perception, attention, memory, preference and motivation.

\section{FILDS OF INTEREST AND EXPERIMENTAL} SET-UP

In the Eye-Tracking \& Vision Lab (www.evalab .unisi.it.) we are working on the design, management, validation and post processing analysis of an EVA system that includes: a multilevel interactive stimulus tool (visual, audio, tactile, joystick, et al), a stimulus database, a gaze analysis function tool and a modeling tool for cognitive and brain function representations

The first study reported here was designed to evaluate the visuo-spatial exploration pattern of patient with cerebellar dysfunction faced with a cognitively demanding task (3).

The cerebellum plays an important role in motor control and learning through representation of an internal model that may reproduce dynamic properties of the body. These models allow exact control of body movements, even in the absence of sensory feed-back. Analogously, it seems that the cerebellum encodes the internal model, reproducing major behaviour and cognitive patterns.

Several studies on patients with disorders of the cerebellum have documented alterations in specific cognitive performances; it is well known that the cerebellum is implicated in the control of language abilities, presumably creating internal models that automate assembly of words (4). Autism and dyslexia express two models of cerebellar cognitive dysfunction (5). Autism is considered an example in which incapacity to relate to others is caused by alteration of an internal model which encourages emotional and motivational processes. In dyslexia, an incapacity to clip heard words into smaller pieces (bits) causes difficulty in grasping/apprehending specific phenomena and associations. This deficit seems to stem from a small brain capacity for filtering irrelevant output or perceptual noise (6). Patients with developmental dyslexia have disorders of the frontal and temporal lobes, the magnocellular visual system and cerebellum. Using a test for dyslexia, we found a specific visual pattern, characterized by continuous re-fixations of letters previously encompassed by gaze, that suggests altered working memory in these subjects.

In another group of patients with cerebellar ataxia (genetically determined) we observed meaningful alterations in complex cognitive exploration tasks, such as the modified trail-making test, a test that explores the capacity to plan, associate, shift attention and organize paths and logical sequences (7). The 
results of our experiment showed that patients with cerebellar atrophy have reduced exploration times in significant region of interest ROI, associated with an altered sequence of steps; the number of repetitions per step was substantially normal. The results obtained in patients with genetically confirmed cerebellar atrophy showed reduced visual spatial exploration ability with altered planning and shifting capacity but with normal working memory (8)

In another experimental setting we studied attention directing in tasks with emotional content, such as happy and sad facial expressions. We found some indices describing exploration pattern in an interference approach by transcranial magnetic stimulation (TMS) over the dorsolateral prefrontal cortex DLPC. The aim of the study was to find lateralization (DLPC right versus left) in the exploration preferences of expressive facial features. DLPC is known to participate in oculomotor programming control, and inhibiting reflexive saccades furthermore it is suggested that is implicated in emotion control, with specific lateralization as shown in patients with schizophrenia. 26 normal volunteers were enrolled in the study. The study was based on application of GMF2 (a memory guided filtering protocol) designed and validated for the experiment and based on presentation of a sequence of happy and sad faces (epochs 2 and 4) interspersed with faces with a neutral expression. TMS was applied at the beginning of epoch 4. Analysis of the scan path provided indices describing gaze pattern during visual exploration of facial expressions with and without TMS (fig. 4). In particular, the expression extraction index (EEI) gave a good representation of the pattern of exploration (9). TMS over the left DPLC was associated with an increase in EEI. Although these results need further investigation they suggests that the right prefrontal cortex has a role in emotion control.

\section{REFERENCES}

1)Leigh $R$ J, Zee D S. The neurology of eye movements. 4th ed. Oxford University Press.:598-725. 2006

2) Martine-Condez S, Macknik S, Hubel DH. The role of fixational eye movements in visual perception. Nat Neurosci5: 229240. (2004)

3)Ito M. Bases and implications of learning in the cerebellum. Adaptative control and internal model mechanism. Prog Brain Res 148: 95-109. 2006

4)Ito M. Control of mental activities by internal models in the cerebellum. Nat Neurosci April:304-313. 2008

5) Lee $M$ et al Nicotinic receptor abnormalities in the cerebellar cortex in autism. Brain125: 1483-1495. 2002

6) Sperling AJ, Lu ZL, Manis FR, Seidenberg MS. Deficits in perceptual noise exclusion in perceptual dyslexia. Nat Neurosci. 8: 862-863. 2005

7)Bowie C, Harvey P. Administration and interpretation of the Trail Macking Test. Nature prot. 1;5:22772281. 2006

8)Lepsien J, Nobre A. Cognitive control of attention in human brain: Insights from orientation to mental representations.1105:20-31. 2006

9) Gilchrist ID Harvey M. Refixation frequency and memory mechanisms in visual search. Curr Biology 10:1209-1212. 2000

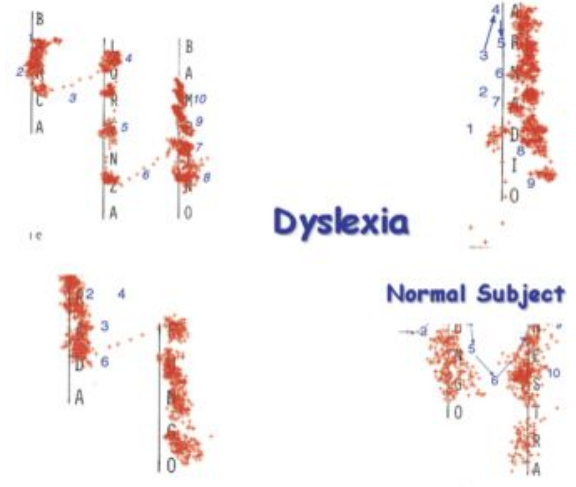

Fig.l:

Visual exploration pattern (scan-path sequenze of fixations and saccades) in patients with Dislexia

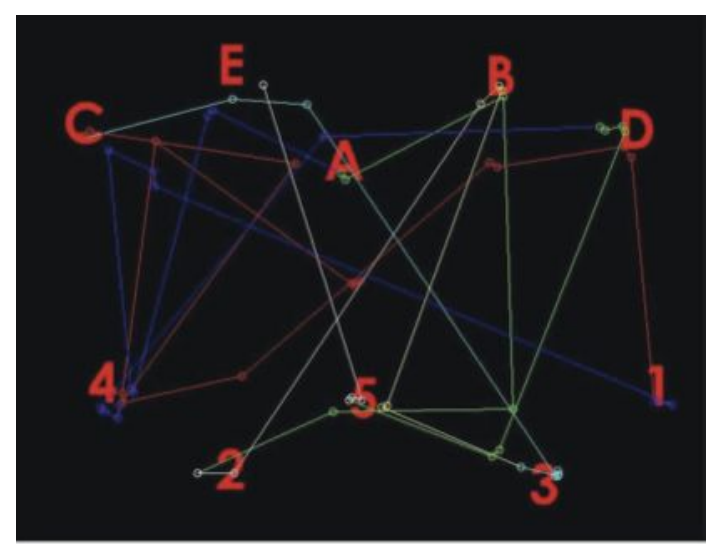

Fig.2:

Visual exploration pattern during a modified trial making test TMT in cerebellar patients (the eye tracking study. Scan-path visualization)

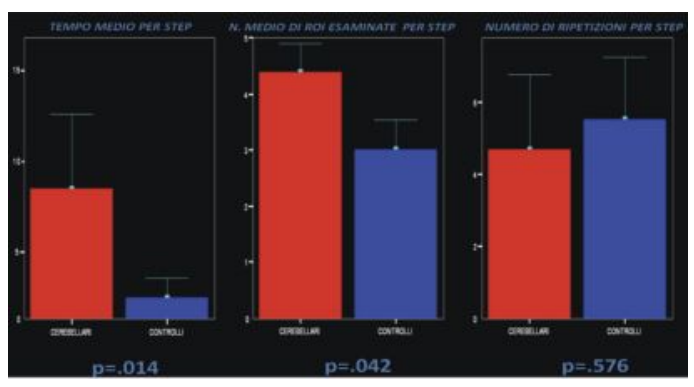

Fig.3:

Cerebellar subject vs controls

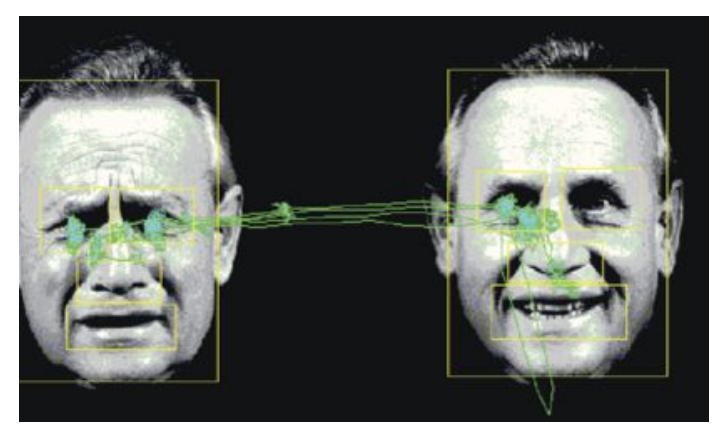

Fig.4:

Visual exploration of emotional facial features ROIs 